$x=m a b c f g h p q r, y=m g h(a f)^{2} p^{3}, z=m c a(b g)^{2} q^{3}, w=m b f(c h)^{2} r^{3}$, where the parameters $m, \cdots, r$ may be restricted by the G.C.D. conditions

$$
\begin{aligned}
& 1=(a, f)=(b, g)=(c, h), \\
& 1=(a f p, b c q r)=(b g q, h f r p)=(c h r, a g p q) .
\end{aligned}
$$

The most immediate application of this is to the solution of $x^{3}+f(y, z, w)=0$, where $f(y, z, w)$ is any ternary cubic factorable into 3 linear, homogeneous factors whose determinant is \pm 1 . The solution can be written down from the above.

California Institute of Technology

\title{
A NOTE ON THE FOUR-POINT PROPERTY
}

BY L. M. BLUMENTHAL

1. Introduction. It has been pointed out by Menger that a metric space may be defined as a semi-metric space each three points of which is congruent to three points of a euclidean plane.

In a recent article, W. A. Wilson has considered a metric space that has the property that any $n$ points of the space can be imbedded in a euclidean $(n-1)$-dimensional space.* Such a space is said to have the $n$-point property. An investigation of such spaces led Wilson to two important theorems. He shows (1) that if a convex, externally convex, and complete space has the four-point property, then the space has the $n$-point property for every integer $n$, and (2) that a convex, externally convex, complete, and separable space which has the four-point property is congruent with some euclidean space or with Hilbert space. It follows from the first theorem that pseudo-euclidean sets do not exist in a space satisfying the hypotheses of the theorem. This fact is of importance in connection with the problem of determining surfaces in which pseudo-euclidean $n$-tuples can be imbedded, for $n$ greater than four. The second theorem, obtained by applying the first one to a well known theorem of

* W. A. Wilson, A relation between metric and euclidean spaces, American Journal of Mathematics, vol. 54 (1932), pp. 505-517. 
Menger,* shows the great significance of the four-point property in the characterization of euclidean spaces among general semi-metric spaces.

These interesting results focus attention upon the four-point property. It is the purpose of this note to obtain a new formulation of the necessary and sufficient condition that four points of a metric space be congruent with four points of a euclidean space. While it is of interest to note that the condition turns out to be similar to the triangle axiom, the most significant feature of the new result is that it serves to relate the theory of the euclidean $n$-dimensional space with the spherical space of $n$ dimensions.

2. A New Formulation of the Condition $D_{4} \geqq 0$. If $p_{1}, p_{2}, p_{3}, p_{4}$ are four points of a metric space, it has been shown by Menger in the article referred to that four points of a euclidean space exist congruent to them if and only if

$$
D_{4}=\left|\begin{array}{ccccc}
0 & 1 & 1 & 1 & 1 \\
1 & 0 & \left(p_{1} p_{2}\right)^{2} & \left(p_{1} p_{3}\right)^{2} & \left(p_{1} p_{4}\right)^{2} \\
1 & \left(p_{2} p_{1}\right)^{2} & 0 & \left(p_{2} p_{3}\right)^{2} & \left(p_{2} p_{4}\right)^{2} \\
1 & \left(p_{3} p_{1}\right)^{2} & \left(p_{3} p_{2}\right)^{2} & 0 & \left(p_{3} p_{4}\right)^{2} \\
1 & \left(p_{4} p_{1}\right)^{2} & \left(p_{4} p_{2}\right)^{2} & \left(p_{4} p_{3}\right)^{2} & 0
\end{array}\right| \geqq 0 .
$$

This condition may readily be put in the form $\left|\begin{array}{l}2\left(p_{1} p_{2}\right)^{2},\left(p_{1} p_{2}\right)^{2}+\left(p_{1} p_{3}\right)^{2}-\left(p_{2} p_{3}\right)^{2},\left(p_{1} p_{2}\right)^{2}+\left(p_{1} p_{4}\right)^{2}-\left(p_{2} p_{4}\right)^{2} \\ \left(p_{1} p_{2}\right)^{2}+\left(p_{1} p_{3}\right)^{2}-\left(p_{2} p_{3}\right)^{2}, 2\left(p_{1} p_{3}\right)^{2},\left(p_{1} p_{3}\right)^{2}+\left(p_{1} p_{4}\right)^{2}-\left(p_{3} p_{4}\right)^{2} \\ \left(p_{1} p_{2}\right)^{2}+\left(p_{1} p_{4}\right)^{2}-\left(p_{2} p_{4}\right)^{2},\left(p_{1} p_{3}\right)^{2}+\left(p_{1} p_{4}\right)^{2}-\left(p_{3} p_{4}\right)^{2}, 2\left(p_{1} p_{4}\right)^{2}\end{array}\right| \geqq 0$.

Now each of the four triples contained in the four points is congruent to a planar triple, since the points are, by hypothesis, in a metric space. Applying the law of cosines to the three planar triples congruent to the triples $p_{1}, p_{2}, p_{3} ; p_{1}, p_{2}, p_{4} ; p_{1}, p_{3}$, $p_{4}$, we obtain $\dagger$ from the preceding condition

$$
8\left(p_{1} p_{2}\right)^{2}\left(p_{1} p_{3}\right)^{2}\left(p_{1} p_{4}\right)^{2} \cdot \Delta \geqq 0,
$$

* Menger, New foundation of euclidean geometry, American Journal of Mathematics, vol. 53 (1931), p. 745.

$\dagger$ See R. Baltzer, Die Elemente der Mathematik, 1885, pp. 347-348. 
where

$$
\Delta=\left|\begin{array}{ccc}
1 & \cos \alpha_{23} & \cos \alpha_{24} \\
\cos \alpha_{32} & 1 & \cos \alpha_{34} \\
\cos \alpha_{42} & \cos \alpha_{43} & 1
\end{array}\right|
$$

with $\alpha_{i j}=\alpha_{j i},(i, j=2,3,4)$, where $\alpha_{i j}$ is the angle in the triangle formed by the points $p_{1}^{\prime}, p_{i}^{\prime}, p_{j}^{\prime}$ that are congruent to the points $p_{1}, p_{i}, p_{j}$, opposite the side $p_{i}^{\prime} p_{j}^{\prime}$. Then $0 \leqq \alpha_{i j} \leqq \pi$.

The third-order determinant $\Delta$, whose sign is the sign of the fifth-order determinant $D_{4}$, may be written

$$
\begin{aligned}
\Delta=4 \sin \frac{1}{2}\left(\alpha_{23}+\right. & \left.\alpha_{34}+\alpha_{24}\right) \sin \frac{1}{2}\left(\alpha_{23}+\alpha_{34}-\alpha_{24}\right) \\
& \sin \frac{1}{2}\left(\alpha_{23}-\alpha_{34}+\alpha_{24}\right) \sin \frac{1}{2}\left(-\alpha_{23}+\alpha_{34}+\alpha_{24}\right) .
\end{aligned}
$$

Suppose, now, that $\Delta$ is positive or zero. Then the four points $p_{1}, p_{2}, p_{3}, p_{4}$ are congruent to four points of a euclidean space, and it is clear that the three angles $\alpha_{23}, \alpha_{34}, \alpha_{24}$ satisfy the triangle axiom and have a sum less than or equal to $2 \pi$. Conversely, if the angles $\alpha_{23}, \alpha_{34}, \alpha_{24}$ satisfy the triangle axiom and have a sum less than or equal to $2 \pi$, then the determinant $\Delta$ is positive or zero. This is evident, for if one angle is the sum of the other two, or if the sum of all three angles equals $2 \pi$, then one of the factors in the last expression for $\Delta$ vanishes. Also, if the sum of any two of the angles exceeds the third angle, and the sum of all three is less than $2 \pi$, it is clear that each of the factors of $\Delta$ is positive. Hence the condition $D_{4} \geqq 0$ is seen to be equivalent to the condition that the three angles $\alpha_{23}, \alpha_{34}, \alpha_{24}$ satisfy the triangle axiom and have a sum less than or equal to $2 \pi$.

We say that the angles $\alpha_{23}, \alpha_{34}, \alpha_{24}$ have the point $p_{1}$ for a common vertex, and we state the above result in the following theorem.

TheOREM. Four points of a metric space are congruent to four points of a euclidean space if and only if any three angles having a common vertex satisfy the triangle axiom with a sum not exceeding $2 \pi$.

3. The Spherical Space $S_{2}$. The spherical space $S_{2}$ is formed by the points on the surface of a sphere, where the distance between two points is defined as the length of the shorter arc of the great circle joining them. 
The writer has investigated the problem of characterizing sets of points congruent to points of the $S_{2}$ with radius $r$ ( $r$ spheric sets), as well as sets each four points of which is congruent to four points of a sphere of radius $r$, while the whole set is not congruent to a subset of the sphere (pseudo $r$-spheric sets). It has been shown in a paper not yet published in full* that the necessary and sufficient condition that three points $p_{1}, p_{2}, p_{3}$ of a semi-metric space be congruent with three points of a sphere of radius $r$ is that (1) the distance of each two of the points is not greater than $\pi r$, and (2) the determinant $\Delta_{3}$ is positive or zero, where

$$
\Delta_{3}=\left|\begin{array}{ccc}
1 & \cos \alpha_{12} & \cos \alpha_{13} \\
\cos \alpha_{21} & 1 & \cos \alpha_{23} \\
\cos \alpha_{31} & \cos \alpha_{32} & 1
\end{array}\right|
$$

with $\alpha_{i j}=p_{i} p_{j} / r$ radians, $(i, j=1,2,3)$.

We point out that the second condition is the one investigated in the second section of this paper.

The Rice Institute

* L. M. Blumenthal and G. A. Garrett, The determinantal theory of d-cyclic and pseudo d-cyclic sets of points, this Bulletin, Abstract No. 39-1-46. 